\title{
Semidefinite functions on categories
}

\author{
LÁSZLÓ LOVÁSZ* \\ Institute of Mathematics \\ Eötvös Loránd University, Budapest, Hungary \\ Alexander SchriJver \\ CWI and University of Amsterdam, The Netherlands \\ Submitted: Oct 3, 2008; Accepted: May 25, 2009; Published: May 31, 2009 \\ Mathematics Subject Classification: 05C50 \\ Dedicated to Anders Björner on his $60^{\text {th }}$ birthday.
}

\begin{abstract}
Freedman, Lovász and Schrijver characterized graph parameters that can be represented as the (weighted) number of homomorphisms into a fixed graph. Several extensions of this result have been proved. We use the framework of categories to prove a general theorem of this kind. Similarly as previous resuts, the characterization uses certain infinite matrices, called connection matrices, which are required to be positive semidefinite.
\end{abstract}

\section{Introduction}

For two finite graphs $F$ and $G$, let $\operatorname{hom}(F, G)$ denote the number of homomorphisms $F \rightarrow G$. The definition can be extended to weighted graphs. In [7] graph parameters of the form $\operatorname{hom}(\cdot, G)$, defined on finite multigraphs, were characterized, where $G$ is a fixed weighted graph. Several variants of this result have been obtained, characterizing graph parameters $\operatorname{hom}(\cdot, G)$ where all nodeweights of $G$ are 1 [16], such graph parameters defined on simple graphs [13] etc. These characterizations involve certain infinite matrices, called connection matrices, which are required to be positive semidefinite and to satisfy a condition on their rank. The results can be extended to directed graphs, hypergraphs etc.

The goal of this paper is to use the framework of categories to prove a general theorem of this kind. Let $\mathcal{C}$ be a category. We need to assume that it satisfies a number of natural conditions C1. 4 below, but for the statement of the main theorem we only need that it is locally finite, it has pullbacks, and it contains a terminal object $t$. In particular, every

\footnotetext{
${ }^{*}$ Research sponsored by OTKA Grant No. 67867.
} 
two objects $a$ and $b$ have a direct product $a \times b$. We denote by $\mathcal{C}(a, b)$ the set of morphisms from $a$ to $b$.

Let $f$ be a real valued function defined on the objects, invariant under isomorphism. We say that $f$ is multiplicative, if $f(a \times b)=f(a) f(b)$ for any two objects $a$ and $b$. For every object $a$, we define a (possibly infinite) symmetric matrix $N(f, a)$, whose rows and columns are indexed by the morphisms into $a$, and whose entry in row $\alpha$ and column $\beta$ is $f(d)$, where $d$ is the object where the pullback of $(\alpha, \beta)$ starts (this is well defined up to isomorphism).

Theorem 1 Let $\mathcal{C}$ be a category satisfying conditions Q11-44 below. Let $f$ be a function defined on the objects, invariant under isomorphism. Then $f=|\mathcal{C}(b,)$.$| for some object b$ if and only if the following conditions are fulfilled: (F1) $f(t)=1,(\mathrm{~F} 2) f$ is multiplicative, and (F3) $N(f, a)$ is positive semidefinite for every object a.

We note that if there is a monomorphism from $a$ to $b$, then $N(f, a)$ is a submatrix of $N(f, b)$. Thus it would be enough to require the semidefiniteness condition for an appropriate subset $K$ of objects such that every object has a monomorphism into some $k \in K$ (we call such a set $K$ cofinal). Since $a \times t$ is isomorphic with $a$, condition (F1) follows from (F2) unless $f$ is identically 0 .

Let us mention a corollary.

Corollary 2 Conditions (F1)-(F3) of the theorem imply that (a) the values of $f$ are non-negative integers, $(b)$ the rank of $N(f, a)$ is finite.

Part (a) contrasts this result with the results of [7, 16], where (thanks to the weights) the function values can be arbitrary. An analogue of (b) must be imposed as a condition e.g. in the characterization in [7, while in this setup it follows from the other assumptions.

\section{Preliminaries}

\subsection{Conditions on the category}

Let $\mathcal{C}$ be a category (for basic definitions and facts, see e.g. [1]). For two objects $a, b \in$ $\mathrm{Ob}(\mathcal{C})$, we denote by $\mathcal{C}(a, b)$ the set of morphisms $a \rightarrow b$. We denote the composition of two morphisms $\alpha \in \mathcal{C}(a, b)$ and $\beta \in \mathcal{C}(b, c)$ by $\alpha \beta$. For $\alpha \in \mathcal{C}(a, b)$, we set $T(\alpha):=a$ (tail of $\alpha$ ) and $H(\alpha):=b$ (head of $\alpha$ ). Let $\mathcal{C}_{a}$ denote the set of morphisms with $H(\alpha)=a$. We denote by $\mathcal{C}^{\text {mon }}(a, b)$ and by $\mathcal{C}_{a}^{\text {mon }}$ the set of monomorphisms in $\mathcal{C}(a, b)$ and $\mathcal{C}_{a}$, respectively.

We make the following assumptions about our category.

C1 $\mathcal{C}$ is locally finite, i.e., $\mathcal{C}(a, b)$ is finite for all $a, b$.

C2 (a) $\mathcal{C}$ has pullbacks.

(b) $\mathcal{C}$ has a terminal object $t$, into which every object has a unique morphism (which can be thought of as the pullback of the empty set of morphisms). 
C3 Every morphism is the product of an epimorphism and a monomorphism.

C4 The category has an object such that the set of its direct powers is cofinal (we call such an object a generator).

For every object $a$, we introduce an equivalence relation on $\mathcal{C}_{a}$ by $\alpha \equiv \beta$ if and only if $\beta=\gamma \alpha$ for some isomorphism $\gamma$. We say that $\alpha$ and $\beta$ are left-isomorphic. We denote by $[\alpha]$ the equivalence class of $\alpha$, and by $\widehat{\mathcal{C}}_{a}$, the set of equivalence classes in $\mathcal{C}_{a}$.

Recall that for two morphisms $\alpha \in \mathcal{C}(a, c)$ and $\beta \in \mathcal{C}(b, c)$, a pair of morphisms $\alpha^{\prime} \in \mathcal{C}(d, a)$ and $\beta^{\prime} \in \mathcal{C}(d, b)$ is called a pullback of $(\alpha, \beta)$ if $\alpha^{\prime} \alpha=\beta^{\prime} \beta$, and whenever $\xi \in \mathcal{C}(e, a)$ and $\zeta \in \mathcal{C}(e, b)$ are two morphisms such that $\xi \alpha=\zeta \beta$, then there is a unique morphism $\eta \in \mathcal{C}(e, d)$ such that $\eta \alpha^{\prime}=\xi$ and $\eta \beta^{\prime}=\zeta$. We also call $\alpha^{\prime}$ a pullback of $\beta$ along $\alpha$.

In terms of $\alpha$ and $\beta$, we write

$$
\alpha \beta^{*}:=\beta^{\prime}, \quad \beta \alpha^{*}:=\alpha^{\prime}, \quad \alpha \times \beta:=\alpha^{\prime} \alpha=\beta^{\prime} \beta .
$$

(This strange notation will be convenient later on.)

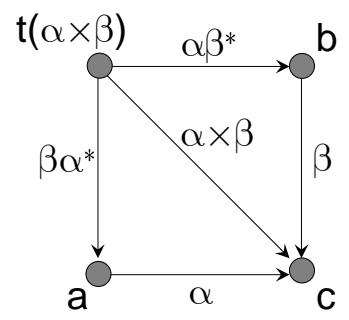

Figure 1: Pullbacks and product

It is well known and easy to check that for $\alpha, \beta \in \mathcal{C}_{a},\left[\beta \alpha^{*}\right]$ only depends on $[\beta]$, and $[\alpha \times \beta]$ only depends on $[\alpha]$ and $[\beta]$. The object $T(\alpha \times \beta)$ is determined up to isomorphism. Furthermore, if $\left[\alpha_{1}\right]=\left[\alpha_{2}\right]$, then $\left[\alpha_{1} \beta^{*}\right]=\left[\alpha_{2} \beta^{*}\right]$ and $\left[\beta \times \alpha_{1}\right]=\left[\beta \times \alpha_{2}\right]$. So the operation $x$ is well defined on equivalence classes of morphisms. It is also clear that if $\alpha_{1}, \alpha_{2} \in \mathcal{C}(a, b), \varphi \in \mathcal{C}(b, c)$, and $\left[\alpha_{1}\right]=\left[\alpha_{2}\right]$, then $\left[\alpha_{1} \varphi\right]=\left[\alpha_{2} \varphi\right]$. This defines $[\alpha] \times[\beta]:=[\alpha \times \beta]$. It is easy to see that the operation $\times$ on $\widehat{\mathcal{C}}_{a}$ is associative and commutative.

We say that the category has pullbacks (condition C2(a)) if every pair of morphisms into the same object has a pullback. A direct product $a \times b$ of two objects is any object of the form $T(\alpha \times \beta)$, where $\alpha$ and $\beta$ are the unique morphisms of $a$ and $b$ into the terminal object $t$. This is uniquely determined up to isomorphism.

\section{$2.2 \quad$ Examples}

Example 1 The category of finite simple graphs with loops (where morphisms are homomorphisms, i.e., adjacency-preserving maps) satisfies these assumptions. Conditions C11 and C3 are trivial. 
The terminal object in C2(b) is the single node with a loop, while any complete graph on 2 or more nodes with loops can serve as a generator object as in C4. To construct the pullback of two homomorphisms $\alpha: a \rightarrow c$ and $\beta: b \rightarrow c$, take the direct (categorial) product $d$ of the two graphs $a$ and $b$, together with its projections $\pi_{a}$ and $\pi_{b}$ onto $a$ and $b$, respectively, and take the subgraph $d^{\prime}$ of $d$ induced by those nodes $v$ for which $\left(\pi_{a} \alpha\right)(v)=\left(\pi_{b} \beta\right)(v)$, together with the restrictions of $\pi_{a}$ and $\pi_{b}$ onto $d^{\prime}$.

The cofinal set mentioned in the remark after the Theorem can be the set of all complete graphs with loops at all nodes, in which case the conditions of Theorem 1 are exactly the conditions given in [1].

Example 2 Reversing the arrows in the category of finite simple graphs with loops (Example 11) gives another category satisfying the assumptions.

Conditions C[1 and C3 are again trivial. The terminal object in C2(b) is the empty graph, a generator object is the single node without a loop.

In this dual setting, we have to construct the pushout of two homomorphisms $\alpha: c \rightarrow$ $a)$ and $\beta: c \rightarrow b$ ). This can be done by taking the disjoint union of the two graphs $a$ and $b$, and identifying those nodes that are the images of one and the same node of $c$. This is just the construction of the connection matrix given in [11. The cofinal set mentioned in the remark after the Theorem can be the set of all graphs with no edges, in which case the conditions of Theorem 1 are exactly the conditions given in [1] for this dual setting.

We note that the conditions are very similar to those in [7], except that there the graphs cannot have loops and the matrices are indexed by monomorphisms only. As a consequence, the characterization concerns homomorphism numbers into weighted graphs, which is an extension not considered in this paper.

These examples can be extended to simplicial maps between simplicial complexes, homomorphisms between directed graphs, hypergraphs, etc.

\subsection{Some simple properties of the category}

We state some easy consequences of these assumptions. It is easy to see that condition C1] (local finiteness) implies:

Lemma 3 (a) Every monomorphism [epimorphism] $\mu \in \mathcal{C}(a, a)$ is an isomorphism.

(b) If both $\mathcal{C}(a, b)$ and $\mathcal{C}(b, a)$ contain monomorphisms [epimorphisms], then a is isomorphic to $b$.

Another consequence of condition C1 is that generator objects have alternative characterizations.

Lemma 4 For every object $g$, the following are equivalent.

(i) $g$ is a generator.

(ii) Every object a has a monomorphism into the direct power $g^{|\mathcal{C}(a, g)|}$.

(iii) For any two objects $a, b$ and any two different morphisms $\alpha, \beta \in \mathcal{C}(b, a)$ there is a morphism $\eta \in \mathcal{C}(a, g)$ such that $\alpha \eta \neq \beta \eta$. 

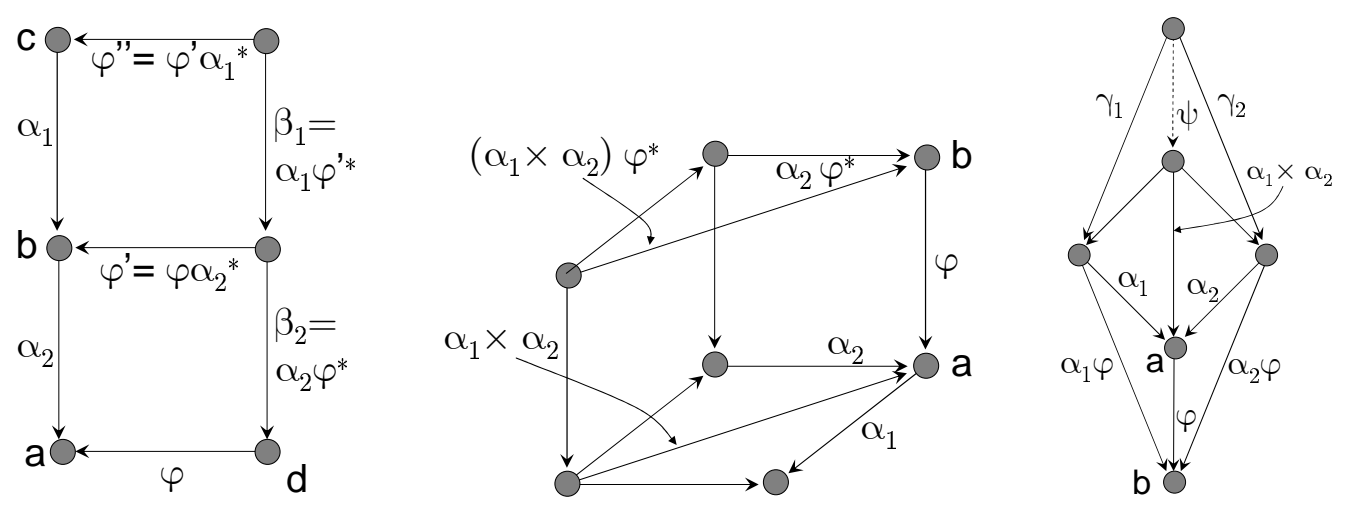

Figure 2: Identities (a), (b) and (c) in Lemma 6 .

(Condition (iii) is the more standard definition of a generator in a category.)

Proof. Clearly (ii) is a sharper form of (i), so it suffices to prove that (i) $\Rightarrow$ (iii) and (iii) $\Rightarrow$ (ii).

(i) $\Rightarrow$ (iii). We know that there is a $k$ such that $a$ has a monomorphism $\xi$ into $g^{k}$. Then $\alpha \xi \neq \beta \xi$. Let $\pi_{1}, \ldots, \pi_{k}$ be the canonical morphisms of $g^{k}$ into $g$, then by the definition of pullback, there is an $i \in\{1, \ldots, k\}$ such that $\alpha \xi \pi_{i} \neq \beta \xi \pi_{i}$. So we can take $\eta=\xi \pi_{i}$.

(iii) $\Rightarrow$ (ii). Let $\mathcal{C}(a, g)=\left\{\varphi_{1}, \ldots, \varphi_{k}\right\}$. By the definition of pullbacks, there is a map $\xi \in \mathcal{C}\left(a, g^{k}\right)$ such that $\xi \pi_{i}=\varphi_{i}$ for $i \in\{1, \ldots, k\}$. We claim that $\xi$ is a monomorphism. Indeed, for any two different morphisms $\alpha, \beta \in \mathcal{C}(b, a)$ there is an $i$ such that $\alpha \varphi_{i} \neq \beta \varphi_{i}$, and hence $\alpha \xi \neq \beta \xi$.

The following lemma is easy to verify:

Lemma 5 Let $\alpha_{1} \in \mathcal{C}(c, b), \alpha_{2} \in \mathcal{C}(b, a)$ and $\varphi \in \mathcal{C}(d, a)$. Let $\left(\varphi^{\prime}, \beta_{2}\right)$ be a pullback of $\left(\alpha_{2}, \varphi\right)$, and let $\left(\varphi^{\prime \prime}, \beta_{1}\right)$ be a pullback of $\left(\alpha_{1}\right.$, varphi' $\left.{ }^{\prime}\right)$. Then $\left(\varphi^{\prime \prime}, \beta_{1} \beta_{2}\right)$ is a pullback of $\left(\alpha_{1} \alpha_{2}, \varphi\right)$.

The operations introduced above satisfy some useful identities.

Lemma 6 (a) Let $\alpha_{1} \in \mathcal{C}(c, b), \alpha_{2} \in \mathcal{C}(b, a)$ and $\varphi \in \mathcal{C}(d, a)$. Then $\left[\left(\alpha_{1} \alpha_{2}\right) \varphi^{*}\right]=$ $\left[\left(\alpha_{1}\left(\varphi \alpha_{2}^{*}\right)^{*}\right)\left(\alpha_{2} \varphi^{*}\right)\right]$ and $\left[\varphi\left(\alpha_{1} \alpha_{2}\right)^{*}\right]=\left[\varphi \alpha_{2}^{*} \alpha_{1}^{*}\right]$.

(b) Let $\alpha_{1}, \alpha_{2} \in \mathcal{C}_{a}$ and $\varphi \in \mathcal{C}(b, a)$. Then $\left[\left(\alpha_{1} \times \alpha_{2}\right) \varphi^{*}\right]=\left[\left(\alpha_{1} \varphi^{*}\right) \times\left(\alpha_{2} \varphi^{*}\right)\right]$.

(c) Let $\alpha_{1}, \alpha_{2} \in \mathcal{C}_{a}$ and $\varphi \in \mathcal{C}(a, b)$. If $\varphi$ is a monomorphism, then $\left[\left(\alpha_{1} \times \alpha_{2}\right) \varphi\right]=$ $\left[\left(\alpha_{1} \varphi\right) \times\left(\alpha_{2} \varphi\right)\right]$.

Proof. The identities in (a) just rephrase Lemma 5. For the proof of (b) and (c), we fix a particular choice of the pullbacks, so that we don't have to use [...]. Identity (b) 
follows by the following computation:

$$
\begin{aligned}
& \left(\alpha_{1} \times \alpha_{2}\right) \varphi^{*}=\left(\left(\alpha_{1} \alpha_{2}^{*}\right) \alpha_{2}\right) \varphi^{*}=\left(\alpha_{1} \alpha_{2}^{*}\right)\left(\varphi \alpha_{2}^{*}\right)^{*}\left(\alpha_{2} \varphi^{*}\right) \\
& \text { (using the first identity in }(\mathrm{a})) \\
& =\alpha_{1}\left(\left(\varphi \alpha_{2}^{*}\right) \alpha_{2}\right)^{*}\left(\alpha_{2} \varphi^{*}\right) \\
& \text { (using the second identity in }(\mathrm{a})) \\
& =\alpha_{1}\left(\alpha_{2} \times \varphi\right)^{*} \alpha_{2} \varphi^{*}=\alpha_{1}\left(\left(\alpha_{2} \varphi^{*}\right) \varphi\right)^{*} \alpha_{2} \varphi^{*} \\
& =\left(\alpha_{1} \varphi^{*}\right)\left(\alpha_{2} \varphi^{*}\right)^{*} \alpha_{2} \varphi^{*}=\left(\alpha_{1} \varphi^{*}\right) \times\left(\alpha_{2} \varphi^{*}\right) .
\end{aligned}
$$

To prove (c), let $\alpha_{1} \in \mathcal{C}\left(c_{i}, a\right)$, and $\alpha_{1} \times \alpha_{2} \in \mathcal{C}(d, a)$. We want to prove that $\left(\alpha_{2} \alpha_{1}^{*}, \alpha_{1} \alpha_{2}^{*}\right)$ is a pullback of $\left(\alpha_{1} \varphi, \alpha_{2} \varphi\right)$. Let $e$ be any object and let $\gamma_{i} \in \mathcal{C}\left(e, c_{i}\right)$ be morphisms such that $\gamma_{1} \alpha_{1} \varphi=\gamma_{2} \alpha_{2} \varphi$. Since $\varphi$ is a monomorphism, this implies that $\gamma_{1} \alpha_{1}=\gamma_{2} \alpha_{2}$. Since $\alpha_{1} \alpha_{2}^{*} \in \mathcal{C}\left(d, c_{1}\right)$ and $\alpha_{2} \alpha_{1}^{*} \in \mathcal{C}\left(d, c_{2}\right)$ form a pullback of $\left(\alpha_{1}, \alpha_{2}\right)$, it follows that there is a unique morphism $\psi \in \mathcal{C}(e, d)$ such that $\gamma_{1}=\psi \alpha_{2} \alpha_{1}^{*}$ and $\gamma_{2}=\psi \alpha_{1} \alpha_{2}^{*}$. This proves the assertion.

For each object $a$, the operation $\times$ defines a semigroup on $\widehat{\mathcal{C}}_{a}$. Let $\mathcal{G}_{a}$ denote its semigroup algebra of all formal finite linear combinations of morphisms in $\mathcal{C}_{a}$.

Remark 7 Razborov's "flag algebras" 15] can be defined in our setting as follows. We consider the category of embeddings (injective homomorphisms) between graphs. Fixing a graph $a$ (which Razborov calls a "type"), the morphisms from $a$ correspond to graphs with a specified subgraph isomorphic with $a$ (which Razborov calls a "flag"). The pushout of two such morphisms results in an object obtained by gluing together the two graphs along the image of $a$, which is exactly how Razborov defines the product in flag algebras. So if we reverse the arrows, we get that flag algebras are the algebras $\mathcal{G}_{a}$ in the category of monomorphisms between graphs, with arrows reversed. This is a subalgebra of the algebra $\mathcal{G}_{a}$ defined in terms of all homomorphisms between graphs.

If $\varphi: a \rightarrow b$ is any morphism, then $\alpha \mapsto \alpha \varphi$ extends to a linear map $\mathcal{G}_{a} \rightarrow \mathcal{G}_{b}$, which we denote by $x \mapsto x \varphi$. The map $\beta \mapsto \beta \varphi^{*}$ extends to a linear map $\mathcal{G}_{b} \rightarrow \mathcal{G}_{a}$, which we denote by $x \mapsto x \varphi^{*}$.

Lemma 8 Let $a, b_{1}, b_{2}$ be objects, $\varphi_{i} \in \mathcal{C}\left(b_{i}, a\right)$, and let $\left(\eta_{1}, \eta_{2}\right)$ be a pullback of $\left(\varphi_{1}, \varphi_{2}\right)$. Let $x_{i} \in \mathcal{G}_{b_{i}}$, then $x_{1} \varphi_{1} \times x_{2} \varphi_{2}=\left(x_{1} \eta_{1}^{*} \times x_{2} \eta_{2}^{*}\right)\left(\varphi_{1} \times \varphi_{2}\right)$.

Proof. It suffices to prove this for the case when $x_{i}=\left[\beta_{i}\right]$ for some $\beta_{i} \in \mathcal{C}_{b_{i}}$. Then the equation follows by applying Lemma 6(a) twice.

\section{The easy direction of the proof}

We start with proving the "only if" part of Theorem 1. Suppose that $f=|\mathcal{C}(b,)$.$| for some$ object $b$. Then $f(t)=1$ by the definition of $t$, and $f$ is multiplicative by the definition 
of direct product. To show that $N(f, a)$ is positive semidefinite, consider any $\gamma \in \mathcal{C}(c, a)$ and $\delta \in \mathcal{C}(d, a)$, and let $u=T(\gamma \times \delta)$. Note that, by the definition of pullbacks, $f(u)$ is the number of pairs of morphisms $(\phi, \psi)(\phi \in \mathcal{C}(b, c), \psi \in \mathcal{C}(b, d))$ such that $\phi \gamma=\psi \delta$. Fix any morphism $\mu \in \mathcal{C}(b, a)$, and let $M_{\gamma}^{\mu}$ denote the number of morphisms $\phi \in \mathcal{C}(b, c)$ such that $\phi \gamma=\mu$. Clearly $N(f, a)_{\gamma, \delta}=\sum_{\mu} M_{\gamma}^{\mu} M_{\delta}^{\mu}$, and so the matrix $N$ is the sum $|\mathcal{C}(b, a)|$ positive semidefinite matrices of rank 1 .

Remark 9 The same argument gives a more general semidefiniteness result. Let $\mathcal{C}$ be a locally finite category, and let $c$ and $d$ be two objects. For any two morphisms $\alpha \in$ $\mathcal{C}\left(a, a^{\prime}\right)$ and $\beta \in \mathcal{C}\left(b, b^{\prime}\right)$, let $N_{\alpha, \beta}$ denote the number of 4-tuples of morphisms $(\phi, \psi, \mu, \nu)$ $\left(\phi \in \mathcal{C}(c, a), \psi \in \mathcal{C}(c, b), \mu \in \mathcal{C}\left(a^{\prime}, d\right), \nu \in \mathcal{C}\left(b^{\prime}, d\right)\right)$ such that $\phi \alpha \mu=\psi \beta \nu$. Then the matrix $N=\left(N_{\alpha, \beta}\right)$, where $\alpha$ and $\beta$ range over all morphisms of the category, is positive semidefinite.

\section{Factoring by $f$}

Let $f: \mathcal{C} \rightarrow \mathbb{R}$ be any function invariant under isomorphism. It will be convenient to extend it to morphisms, and define $f(\varphi)=f(T(\varphi))$. Clearly, this extension is invariant under left-isomorphism of morphisms. We can extend $f$ to the algebras $\mathcal{G}_{a}$ linearly. It follows from the definition that for $x \in \mathcal{G}_{a}$ and $\varphi \in \mathcal{C}(a, b)$ we have $f(x \varphi)=f(x)$.

For $\alpha, \beta \in \mathcal{C}_{a}$, we define

$$
\langle\alpha, \beta\rangle=f(\alpha \times \beta),
$$

which defines a (generally indefinite) inner product on $\mathcal{G}_{a}$. Lemma 6(a) implies that for $x \in \mathcal{G}_{a}, y \in \mathcal{G}_{b}$ and $\varphi \in \mathcal{C}(a, b)$ the following identity holds:

$$
\langle x \varphi, y\rangle=\left\langle x, y \varphi^{*}\right\rangle
$$

(which justifies the notation $\varphi^{*}$ ). Furthermore, Lemma G(c) implies that if $\varphi \in \mathcal{C}(a, b)$ is a monomorphism, then for $x, y \in \mathcal{C}_{a}$,

$$
\langle x \varphi, y \varphi\rangle=f(x \varphi \times y \varphi)=f((x \times y) \varphi)=f(x \times y)=\langle x, y\rangle .
$$

It also follows from the definition and the associativity of the product $\times$ that

$$
\langle\alpha \times \beta, \gamma\rangle=f(\alpha \times \beta \times \gamma)=\langle\alpha, \beta \times \gamma\rangle
$$

for all $\alpha, \beta, \gamma$ in $\mathcal{C}_{a}$. This extends linearly to the identity

$$
\langle x \times y, z\rangle=\langle x, y \times z\rangle
$$

for all $x, y, z \in \mathcal{G}_{a}$.

Let

$$
\mathcal{N}_{a}=\left\{x \in \mathcal{G}_{a}:\langle x, y\rangle=0 \text { for all } y \in \mathcal{G}_{a}\right\},
$$

then $\mathcal{N}_{a}$ is an ideal in the algebra $\mathcal{G}_{a}$, since if $x \in \mathcal{N}_{a}$, then by (4), we have for all $y, z \in \mathcal{G}_{a}$, $\langle x \times y, z\rangle=\langle x, y \times z\rangle=0$, and hence $x \times y \in \mathcal{N}_{a}$. So we can form the factor $\mathcal{A}_{a}=\mathcal{G}_{a} / \mathcal{N}_{a}$, which is an associative and commutative algebra with a (possibly indefinite) inner product $\langle.,$.$\rangle . The coset \mathcal{N}_{a}+\mathrm{id}_{a}$ is an identity element in $\mathcal{A}_{a}$, which we denote by $\mathbf{1}_{a}$. 
Lemma 10 Let $\varphi \in \mathcal{C}(a, b)$.

(a) If $x \in \mathcal{N}_{a}$ then $x \varphi \in \mathcal{N}_{b}$.

(b) If $y \in \mathcal{N}_{b}$ then $y \varphi^{*} \in \mathcal{N}_{a}$.

(c) If $\varphi$ is a monomorphism, then $x \varphi \in \mathcal{N}_{b}$ implies that $x \in \mathcal{N}_{a}$.

Proof. (a) To prove that $x \varphi \in \mathcal{N}_{b}$, we want to prove that $\langle x \varphi, y\rangle=0$ for all $y \in \mathcal{G}_{b}$. By (11), $\langle x \varphi, y\rangle=\left\langle x, y \varphi^{*}\right\rangle$, which is 0 as $x \in \mathcal{N}_{a}$.

(b) To prove that $y \varphi^{*} \in \mathcal{N}_{a}$, we want to prove that $\left\langle y \varphi^{*}, x\right\rangle=0$ for all $x \in \mathcal{G}_{a}$. Similarly as before, $\left\langle y \varphi^{*}, x\right\rangle=\langle y, x \varphi\rangle=0$ as $y \in \mathcal{N}_{b}$.

(c) Assume that $x \varphi \in \mathcal{N}_{b}$ for some $x \in \mathcal{G}_{a}$. Then $\langle x \varphi, y\rangle=0$ for every $y \in \mathcal{G}_{b}$, in particular, $\langle x \varphi, z \varphi\rangle=0$ for every $z \in \mathcal{G}_{a}$. Then by (2), $\langle x, z\rangle=0$ for every $z \in \mathcal{G}_{a}$, and so $x \in \mathcal{N}_{a}$.

Corollary 11 (a) The maps $x \mapsto x \varphi$ and $y \mapsto y \varphi^{*}$ induce linear maps from $\mathcal{A}_{a} \rightarrow \mathcal{A}_{b}$ and $\mathcal{A}_{b} \rightarrow \mathcal{A}_{a}$, respectively.

(b) The map $y \mapsto y \varphi^{*}$ induces an algebra homomorphism.

(c) If $\varphi$ is a monomorphism, then the map $x \mapsto x \varphi$ induces an injective algebra homomorphism.

We need some simple facts about inner products in direct products.

Lemma 12 Let $a, b_{1}, b_{2}$ be objects, $\varphi_{i} \in \mathcal{C}\left(b_{i}, a\right)$, and let $\left(\eta_{1}, \eta_{2}\right)$ be a pullback of $\left(\varphi_{2}, \varphi_{2}\right)$. Let $x_{i} \in \mathcal{G}_{b_{i}}$, then

$$
\left\langle x_{1} \eta_{1}^{*}, x_{2} \eta_{2}^{*}\right\rangle=\left\langle x_{1} \varphi_{1}, x_{2} \varphi_{2}\right\rangle
$$

In particular if $a=t$, then

$$
\left\langle x_{1} \eta_{1}^{*}, x_{2} \eta_{2}^{*}\right\rangle=f\left(x_{1}\right) f\left(x_{2}\right)
$$

and for $x_{i}, y_{i} \in \mathcal{G}_{b_{i}}$,

$$
\left\langle x_{1} \eta_{1}^{*} \times x_{2} \eta_{2}^{*}, y_{1} \eta_{1}^{*} \times y_{2} \eta_{2}^{*}\right\rangle=f\left(x_{1} \times y_{1}\right) f\left(x_{2} \times y_{2}\right) .
$$

Proof. The first assertion follows from Lemma 8

$$
\begin{aligned}
\left\langle x_{1} \varphi_{1}, x_{2} \varphi_{2}\right\rangle & =f\left(x_{1} \varphi_{1} \times x_{2} \varphi_{2}\right)=f\left(\left(x_{1} \eta_{1}^{*} \times x_{2} \eta_{2}^{*}\right)\left(\varphi_{1} \times \varphi_{2}\right)\right) \\
& =f\left(x_{1} \eta_{1}^{*} \times x_{2} \eta_{2}^{*}\right)=\left\langle x_{1} \eta_{1}^{*}, x_{2} \eta_{2}^{*}\right\rangle .
\end{aligned}
$$

For the second assertion, it suffices to note that if $a=t$, then by the multiplicativity of $f$,

$$
f\left(x_{1} \varphi_{1} \times x_{2} \varphi_{2}\right)=f\left(x_{1} \varphi_{1}\right) f\left(x_{2} \varphi_{2}\right)=f\left(x_{1}\right) f\left(x_{2}\right),
$$

and using that $\eta_{i}^{*}$ is an algebra homomorphism,

$$
\begin{aligned}
\left\langle x_{1} \eta_{1}^{*} \times x_{2} \eta_{2}^{*},\right. & \left.y_{1} \eta_{1}^{*} \times y_{2} \eta_{2}^{*}\right\rangle=f\left(x_{1} \eta_{1}^{*} \times x_{2} \eta_{2}^{*} \times y_{1} \eta_{1}^{*} \times y_{2} \eta_{2}^{*}\right) \\
= & f\left(x_{1} \eta_{1}^{*} \times y_{1} \eta_{1}^{*} \times x_{2} \eta_{2}^{*} \times y_{2} \eta_{2}^{*}\right)=f\left(\left(x_{1} \times y_{1}\right) \eta_{1}^{*} \times\left(x_{2} \times y_{2}\right) \eta_{2}^{*}\right) \\
= & f\left(x_{1} \times y_{1}\right) f\left(x_{2} \times y_{2}\right) .
\end{aligned}
$$




\section{Semidefiniteness}

To use the hypothesis about semidefiniteness, we start with a simpe observation:

Lemma 13 The inner product $\langle.,$.$\rangle is positive semidefinite on \mathcal{G}_{a}$ if and only if the matrix $N(f, a)$ is positive semidefinite.

Proof. Let $x=\sum_{\alpha} x \alpha \in \mathcal{G}_{a}$. We can also think of $x$ as a column vector indexed by morphisms $\alpha \in \mathcal{C}_{a}$. Then

$$
\begin{aligned}
\langle x, x\rangle & =\sum_{\alpha, \beta}\langle\alpha, \beta\rangle x_{\alpha} x_{\beta}=\sum_{\alpha, \beta} f(\alpha \times \beta) x_{\alpha} x_{\beta} \\
& =\sum_{\alpha, \beta} N(f, a)_{\alpha, \beta} x_{\alpha} x_{\beta}=x^{\top} N(f, a) x .
\end{aligned}
$$

This is nonnegative for all $x \in \mathcal{G}_{a}$ if and only if $N(f, a)$ is positive semidefinite.

From now on we assume that all of the matrices $N(f, a)$ are positive semidefinite, and so the inner product $\langle.,$.$\rangle is positive semidefinite on every \mathcal{G}_{a}$ and then also on every $\mathcal{A}_{a}$.

Lemma 14 The algebra $\mathcal{A}_{a}$ is finite dimensional and $\operatorname{dim}\left(\mathcal{A}_{a}\right) \leq f(a)$.

(The proof, which is an extension of Szegedy's argument in [17], only uses that $N(f, a \times a)$ is positive semidefinite.)

Proof. Let $\pi_{1}, \pi_{2} \in \mathcal{C}(a \times a, a)$ be the canonical projections of $a \times a$ onto $a$. There is a unique morphism $\varphi \in \mathcal{C}(a, a \times a)$ (the "diagonal embedding") such that $\varphi \pi_{1}=\varphi \pi_{2}=\mathrm{id}_{a}$.

Let $e_{1}, \ldots, e_{N}$ be mutually orthogonal unit vectors in $\mathcal{A}_{a}$. Both assertions will follow if we prove that $N \leq f(a)$.

Let

$$
x=\sum_{i=1}^{N}\left(e_{i} \pi_{1}^{*} \times e_{i} \pi_{2}^{*}\right)-[\varphi] .
$$

Then

$$
\begin{aligned}
\langle x, x\rangle & =\sum_{i=1}^{N}\left\langle e_{i} \pi_{1}^{*} \times e_{i} \pi_{2}^{*}, e_{i} \pi_{1}^{*} \times e_{i} \pi_{2}^{*}\right\rangle+2 \sum_{i<j}\left\langle e_{i} \pi_{1}^{*} \times e_{i} \pi_{2}^{*}, e_{j} \pi_{1}^{*} \times e_{j} \pi_{2}^{*}\right\rangle \\
& -2 \sum_{i=1}^{N}\left\langle e_{i} \pi_{1}^{*} \times e_{i} \pi_{2}^{*}, \varphi\right\rangle+\langle\varphi, \varphi\rangle .
\end{aligned}
$$

Here using Lemma 12

$$
\left\langle e_{i} \pi_{1}^{*} \times e_{i} \pi_{2}^{*}, e_{i} \pi_{1}^{*} \times e_{i} \pi_{2}^{*}\right\rangle=f\left(e_{i} \times e_{i}\right)^{2}=\left\langle e_{i}, e_{i}\right\rangle^{2}=1 .
$$

Similarly,

$$
\left\langle e_{i} \pi_{1}^{*} \times e_{i} \pi_{2}^{*}, e_{j} \pi_{1}^{*} \times e_{j} \pi_{2}^{*}\right\rangle=\left\langle e_{i}, e_{j}\right\rangle^{2}=0 .
$$


Furthermore, using that

$$
e_{i} \pi_{2}^{*} \times \varphi=\left(e_{i} \pi_{2}^{*} \varphi^{*}\right) \varphi=\left(e_{i}\left(\varphi \pi_{2}\right)^{*}\right) \varphi=e_{i} \varphi
$$

we have

$$
\left\langle e_{i} \pi_{1}^{*} \times e_{i} \pi_{2}^{*}, \varphi\right\rangle=\left\langle e_{i} \pi_{1}^{*}, e_{i} \pi_{2}^{*} \times \varphi\right\rangle=\left\langle e_{i} \pi_{1}^{*}, e_{i} \varphi\right\rangle=\left\langle e_{i}, e_{i} \varphi \pi_{1}\right\rangle=\left\langle e_{i}, e_{i}\right\rangle=1 .
$$

Since $[\varphi]$ is an idempotent in $\mathcal{G}_{a \times a}$,

$$
\langle\varphi, \varphi\rangle=f(\varphi \times \varphi)=f(\varphi) .
$$

Hence by (15),

$$
\langle x, x\rangle=N+0-2 N+f(\varphi)=f(a)-N .
$$

Since this is nonnegative, the lemma follows.

Since $\langle x \times y, z\rangle=\langle x, y \times z\rangle$ for all $x, y, z \in \mathcal{A}_{a}$, the algebra $\mathcal{A}_{a}$ has a (unique) orthogonal basis $B_{a}$ consisting of idempotents. We call these idempotents the basic idempotents in $\mathcal{A}_{a}$. Every idempotent in $\mathcal{A}_{a}$ is the sum of a subset of $B_{a}$, and in particular

$$
\mathbf{1}_{a}=\sum_{p \in B_{a}} p
$$

Thus the number of idempotents in $\mathcal{A}_{a}$ is finite. Since $[\mu]$ is an idempotent for every monomorphism $\mu \in \mathcal{C}_{a}$, we get an important finiteness property of the category:

Corollary 15 For every object a there are only a finite number of nonequivalent monomorphism into a.

Let $\varphi \in \mathcal{C}(a, b)$. Since $\varphi^{*} \mathcal{A}_{b} \rightarrow \mathcal{A}_{a}$ is an algebra homomorphism, $p \varphi^{*}$ is an idempotent in $\mathcal{A}_{a}$ for any $p \in B_{b}$, and $\mathbf{1}_{b} \varphi^{*}=\mathbf{1}_{a}$. So (6) implies that

$$
\sum_{p \in B_{b}} p \varphi^{*}=\mathbf{1}_{b} \varphi^{*}=\mathbf{1}_{a}=\sum_{q \in B_{a}} q .
$$

For $p \in B_{b}$ and $\varphi \in \mathcal{C}(a, b)$, define

$$
B_{\varphi, p}:=\left\{q \in B_{a}: p \varphi^{*} \times q=q\right\} .
$$

By (7),

$$
p \varphi^{*}=\sum_{q \in B_{\varphi, p}} q .
$$

Lemma 16 Let $p \in B_{b}, q \in B_{a}$, and $\varphi \in \mathcal{C}(a, b)$.

(a) $q \in B_{\varphi, p}$ if and only if

$$
q \varphi=\frac{f(q)}{f(p)} p .
$$

(b) If $q \in B_{\varphi, p}$ and $\varphi$ is a monomorphism, then $q \varphi=p$. 
Note that here $f(q)=f(q \times q)=\langle q, q\rangle>0$ and similarly $f(p)>0$.

Proof. (a) To prove the necessity of the condition, assume that $p^{\prime} \in B_{b} \backslash\{p\}$. Then

$$
\left\langle q \varphi, p^{\prime}\right\rangle=\left\langle q, p^{\prime} \varphi^{*}\right\rangle=0=\left\langle\frac{f(q)}{f(p)} p, p^{\prime}\right\rangle,
$$

since $\left\langle p, p^{\prime}\right\rangle=0$. Moreover,

$$
\langle q \varphi, p\rangle=\left\langle q, p \varphi^{*}\right\rangle=f\left(q \times\left(p \varphi^{*}\right)\right)=f(q)=\left\langle\frac{f(q)}{f(p)} p, p\right\rangle,
$$

since $\langle p, p\rangle=f(p \times p)=f(p)$.

The proof of sufficiency is easy, since $q$ belongs to $B_{\varphi, p^{\prime}}$ for some $p^{\prime} \in B_{b}$, hence $q \varphi=\frac{f(q)}{f\left(p^{\prime}\right)} p^{\prime}$, and so $p=p^{\prime}$.

(b) Notice that $\varphi$ defines an algebra homomorphism from $\mathcal{A}_{a}$ to $\mathcal{A}_{b}$ by Corollary 11](c), and hence using Lemma 6(c),

$$
\frac{f(q)}{f(p)} p=q \varphi=(q \times q) \varphi=(q \varphi) \times(q \varphi)=\left(\frac{f(q)}{f(p)} p\right) \times\left(\frac{f(q)}{f(p)} p\right)=\left(\frac{f(q)}{f(p)}\right)^{2} p,
$$

which implies that $f(q) / f(p)=1$.

\section{Simplified idempotents}

Let $a$ and $b$ be two objects and $x \in \mathcal{A}_{a}, y \in \mathcal{A}_{b}$. We say that $y$ is a simplification of $x$ if there exists a monomorphism $\varphi \in \mathcal{C}(b, a)$ such that $x=y \varphi$. It is clear that a simplification of a simplification is a simplification.

Lemma 17 Every $x \in \mathcal{A}_{a}$ has a unique simplification $y$ such that for every other simplification $z$ of $x, y$ is a simplification of $z$.

Proof. Corollary [15mplies that there is a simplification $y$ of $x$ such that $y$ has no simplification other than itself. We claim that if $z$ is any other simplification of $x$, then $y$ is a simplification of $z$.

Let $y \in \mathcal{A}_{b}$ and $z \in \mathcal{A}_{c}$, and let $\varphi \in \mathcal{C}(b, a)$ and $\psi \in \mathcal{C}(c, a)$ be monomorphisms such that $x=y \varphi=z \psi$. Then

$$
x=y \varphi=\left(\mathbf{1}_{b} \times y\right) \varphi=\mathbf{1}_{b} \varphi \times y \varphi=\mathbf{1}_{b} \varphi \times z \psi .
$$

By Lemma 8, this implies that, setting $d:=T(\varphi \times \psi)$, there is a $u \in \mathcal{A}_{d}$ such that $x=\mathbf{1}_{b} \varphi \times z \psi=u(\varphi \times \psi)$. Since $\varphi \times \psi, \varphi \psi^{*}$ and $\psi \varphi^{*}$ are monomorphisms, this implies that $u$ is a simplification of each of $x, y$ and $z$. So we must have $u=y$, which implies that $y$ is a simplification of $z$ as claimed.

So it follows that every $x \in \mathcal{A}_{a}$ has a "most simplified" version, which we denote by $s(x)$. 
Lemma 18 If $p$ is a basic idempotent, then every simplification of $p$ is a basic idempotent.

Proof. Let $p \in \mathcal{A}_{a}, y \in \mathcal{A}_{b}$ and $p=y \varphi$, where $\varphi \in \mathcal{C}(b, a)$ is a monomorphism. Write $y=\sum_{q \in B_{b}} \lambda_{q} q$. Then $p=y \varphi=\sum_{q \in B_{b}} \lambda_{q} q \varphi$. By Lemma 16, the algebra elements $q \varphi$ are basic idempotents in $\mathcal{A}_{a}$, and so one of them must be equal to $p$. Hence $q \varphi=y \varphi$ for this basic idempotent, and by Corollary 11(c), this implies that $y=q$.

Basic idempotents of the form $s(p)$ will be called simplified.

Lemma 19 Let $p \in B_{a}$ be a simplified basic idempotent, and suppose that $p=p \alpha$ for some $\alpha \in \mathcal{C}(a, a)$. Then $\alpha$ is an isomorphism.

Proof. Write $\alpha=\gamma \delta$, where $\gamma \in \mathcal{C}(a, e)$ is an epimorphism and $\delta \in \mathcal{C}(e, a)$ is a monomorphism. Then $p=p \alpha=(p \gamma) \delta$, and hence by the assumption that $p$ is simplified, it follows that $\delta$ is an isomorphism, and so $\alpha$ is an epimorphism. By Lemma 3, $\alpha$ is an isomorphism.

Lemma 20 Let $p \in B_{a}$ be a simplified basic idempotent, $\varphi \in \mathcal{C}(b, a), q \in B_{\varphi, p}$ and $s(q) \in B_{d}$. Then there is an epimorphism $\eta \in \mathcal{C}(d, a)$ such that $s(q) \in B_{\eta, p}$.

Proof. Let $\mu \in \mathcal{C}(d, b)$ be a monomorphism such that $q=s(q) \mu$. By condition CB, $\mu \varphi$ also factors as $\alpha \beta$, where $\alpha$ is an epimorphism and $\beta$ is a monomorphism. Then

$$
p=\frac{f(p)}{f(q)} q \varphi=\frac{f(p)}{f(q)} s(q) \mu \varphi=\frac{f(p)}{f(q)} s(q) \alpha \beta .
$$

Since $p$ is simplified, this implies that $p=\frac{f(p)}{f(q)} s(q) \alpha \sigma$ for some isomorphism $\sigma$. Setting $\eta=\alpha \sigma$, we get that $s(q) \in B_{\eta, p}$ by Lemma 16.

Lemma 21 If $p \in \mathcal{A}_{a}$ is a simplified basic idempotent, then for every object b,

$$
\operatorname{dim} \mathcal{A}_{b} \geq \frac{\left|\mathcal{C}^{\mathrm{mon}}(a, b)\right|}{\left|\mathcal{C}^{\mathrm{mon}}(a, a)\right|} .
$$

Proof. For every $\varphi \in \mathcal{C}^{\text {mon }}(a, b), p \varphi$ is a basic idempotent in $\mathcal{A}_{b}$. We claim that if $p \varphi=p \psi$, then $[\psi]=[\varphi]$. This will imply that $\mathcal{A}_{b}$ has at least $\left|\mathcal{C}^{\text {mon }}(a, b)\right| /\left|\mathcal{C}^{\text {mon }}(a, a)\right|$ different basic idempotents, which will imply the Lemma.

Let $q:=p \psi=p \varphi$. Let $\sigma=\varphi \times \psi \in \mathcal{C}(c, b)$. By Lemma 8 , there is a $z \in \mathcal{A}_{c}$ such that $p \varphi \times p \psi=z(\varphi \times \psi)$. But $p \varphi \times p \psi=q \times q=q=p \varphi$, and so $z\left(\psi \varphi^{*}\right) \varphi=z(\varphi \times \psi)=q=p \varphi$, whence $z\left(\psi \varphi^{*}\right)=p$ as $\varphi$ is a monomorphism. But $\psi \varphi^{*}$ is also a monomorphism, and since $p$ is simplified, it follows that it is an isomorphism. Similarly, $\varphi \psi^{*}$ is an isomorphism, and hence $\psi=\left(\varphi \psi^{*}\right)^{-1}\left(\psi \varphi^{*}\right) \varphi$, where $\left(\varphi \psi^{*}\right)^{-1}\left(\psi \varphi^{*}\right)$ is an automorphism of $a$. Thus $[\psi]=[\varphi]$.

Our next goal is to prove that the number of simplified basic idempotents is finite. This is where we also use the existence of a generator object $g$. 
Lemma 22 The number of simplified basic idempotents is finite.

Proof. Let $a$ be an object such that $\mathcal{A}_{a}$ has a simplified basic idempotent $p$. Let $m$ be the smallest integer such that $a$ has a monomorphism into $g^{m}$. By Lemma $|\mathcal{C}(a, g)| \geq m$. Hence it follows that $\left|\mathcal{C}\left(a, g^{k}\right)\right| \geq m^{k}$, and so

$$
\left|\mathcal{C}^{\text {mon }}\left(a, g^{k}\right)\right| \geq\left|\mathcal{C}\left(a, g^{k-m}\right)\right|\left|\mathcal{C}^{\text {mon }}\left(a, g^{m}\right)\right| \geq m^{k-m}
$$

for $k \geq m$. Combining with Lemma 21, we get that

$$
\operatorname{dim} \mathcal{A}_{g^{k}} \geq \frac{\left|\mathcal{C}^{\text {mon }}\left(a, g^{k}\right)\right|}{\left|\mathcal{C}^{\text {mon }}(a, a)\right|} \geq \frac{m^{k-m}}{\left|\mathcal{C}^{\text {mon }}(a, a)\right|} .
$$

Using Lemma 14, we get that

$$
m^{k-m} \leq\left|\mathcal{C}^{\text {mon }}(a, a)\right| f\left(g^{k}\right)=\left|\mathcal{C}^{\text {mon }}(a, a)\right| f(g)^{k} .
$$

Letting $k \rightarrow \infty$, we get

$$
m \leq f(g) .
$$

So it follows that $a$ has a monomorphism into $g^{\lfloor f(g)\rfloor}$. Corollary 15 implies that the number of nonisomorphic objects $a$ with this property is finite.

\section{Conclusion}

We say that a simplified basic idempotent $p \in \mathcal{A}_{a}$ is maximal, if whenever $\eta \in \mathcal{C}(b, a)$ is an epimorphism and $q \in B_{\eta, p}$ is a simplified basic idempotent, then $\eta$ is an isomorphism. Lemma 222 implies that there is at least one maximal simplified basic idempotent.

Lemma 23 Let $p \in B_{a}$ be a maximal simplified basic idempotent and $\varphi \in \mathcal{C}(b, a)$. Then

$$
p \varphi^{*}=\sum_{\substack{\psi \in \mathcal{C}(a, b) \\ \psi \varphi=\mathrm{id} a}} p \psi
$$

Proof. Let $q \in B_{b}$. We want to prove that $q \in B_{\varphi, p}$ if and only if $q=p \psi$ for some $\psi \in \mathcal{C}(a, b)$ with $\psi \varphi=\operatorname{id}_{a}$.

If $q=p \psi$ for such a $\psi$, then $q \varphi=p \psi \varphi=p$, and so $q \in B_{\varphi, p}$ by Lemma 16,

Conversely, let $q \in B_{\varphi, p}$, and let $s(q) \in B_{d}$ (Figure 3). By Lemma 20, there is an epimorphism $\eta \in \mathcal{C}(d, a)$ such that $s(q) \in B_{\eta, p}$. By the maximality of $p$, this implies that $\eta$ is an isomorphism, and so $s(q)=p \sigma$ for some isomorphism $\sigma \in \mathcal{C}(a, d)$. It follows that $q=s(q) \mu=p \sigma \mu$ for some monomorphism $\mu \in \mathcal{C}(a, b)$. Then by Lemma 16

$$
p=\frac{f(p)}{f(q)} q \varphi=\frac{f(p)}{f(q)} p \sigma \mu \varphi .
$$

Applying $f$ we see that $f(p)=f(q)$, so $p=q \varphi$. Set $\alpha:=\sigma \mu \varphi$, so $p=p \alpha$. By Lemma 19, $\alpha$ is an isomorphism, and so $\psi=\alpha^{-1} \sigma \mu \in \mathcal{C}(a, b)$ is a monomorphism satisfying $\psi \varphi=\operatorname{id}_{a}$. 


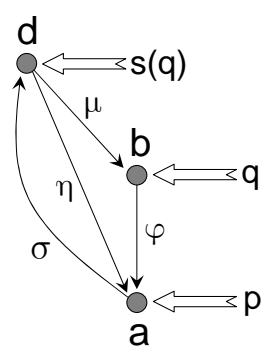

Figure 3: Proof of Lemma 23

Lemma 24 For any two objects $a, b$ and maximal simplified basic idempotent $p \in \mathcal{A}_{a}$,

$$
\sum_{\varphi \in \mathcal{C}(a, b)} p \varphi=f(p) \mathbf{1}_{b}
$$

Proof. By condition $\mathrm{C} 2(\mathrm{~b})$, the category has a terminal object $t$. Let $\mathcal{C}(a, t)=\{\alpha\}$ and $\mathcal{C}(b, t)=\{\beta\}$. Set $\gamma=\beta \alpha^{*}, \delta=\alpha \beta^{*}$, and $c=T(\alpha \times \beta)$.

The algebra $\mathcal{A}_{t}$ is 1-dimensional, which implies that for any $y \in \mathcal{A}_{b}, y \beta$ is a scalar multiple of $\mathbf{1}_{t}$, where $f(y \beta)=f(y)$ and the hypothesis that $f\left(\mathbf{1}_{t}\right)=1$ give the value of the scalar:

$$
y \beta=f(y) \mathbf{1}_{t} .
$$

Furthermore, Lemma 6(b) implies that that

$$
(y \beta) \alpha^{*}=\left(y \delta^{*}\right) \gamma
$$

For each $\varphi \in \mathcal{C}(a, b)$, there is a unique $\psi \in \mathcal{C}(a, T(\alpha \times \beta))$ with $\psi \gamma=\operatorname{id}_{a}$ and $\psi \delta=\varphi$. Hence, with Lemma 23.

$$
\sum_{\varphi \in \mathcal{C}(a, b)} p \varphi=\sum_{\substack{\psi \\ \alpha \psi=\mathrm{id} a}} p \psi \delta=\left(\sum_{\substack{\psi \in \mathcal{C}(a, T(\alpha \times \beta)) \\ \psi \gamma=\mathrm{id} a}} p \psi\right) \delta=\left(p \gamma^{*}\right) \delta .
$$

By (11), (10) and (11), we have for each $y \in \mathcal{A}_{b}$ :

$$
\begin{aligned}
\left\langle y, p \gamma^{*} \delta\right\rangle & =\left\langle y \delta^{*}, p \gamma^{*}\right\rangle=\left\langle y \delta^{*} \gamma, p\right\rangle=\left\langle(y \beta) \alpha^{*}, p\right\rangle \\
& =\langle y \beta, p \alpha\rangle=f(y) f(p)\left\langle\mathbf{1}_{t}, \mathbf{1}_{t}\right\rangle=f(y) f(p)=\left\langle y, f(p) \mathbf{1}_{b}\right\rangle .
\end{aligned}
$$

This implies that $\left(p \gamma^{*}\right) \delta=f(p) \mathbf{1}_{b}$.

We are now ready to prove our main theorem.

Proof of Theorem 1. Let $p \in \mathcal{C}_{a}$ be a maximal simplified basic idempotent. Then for every object $b$, by Lemma 24 .

$$
f(b)=f\left(\mathbf{1}_{b}\right)=\frac{1}{f(p)} \sum_{\varphi \in \mathcal{C}(a, b)} f(p \varphi)=|\mathcal{C}(a, b)| .
$$




\section{Concluding remarks}

Homomorphisms between graphs and their number occur in several other contexts. Which of these results can be extended to categories? Let us discuss some examples.

- Questions of existence of homomorphisms between graphs can often be posed in a very clean form using categorial language (see e.g. [8]).

- Counting homomorphisms has been a main tool in proving cancellation laws for finite relational structures [9]. These results were extended to locally finite categories much in the spirit of this paper [10, 14].

- Counting homomorphisms from fixed graphs into a growing sequence of "large" graphs can be used to define convergence of sequences of graphs and their limit objects [5, 12. Counting homomorphisms from "large" graphs into fixed graphs (usually with weights) connects this subject to statistical physics. Some of these methods have been extended to hypergraphs and other structures [6]. It would be very interesting to extend these notions and results to categories. One can generalize the notions of cut distance and convergence in a rather straightforward way, but it seems to be much harder to generalize some of the basic proofs, and to find interesting special categories to which the general results would apply.

- The set of homomorphisms between two graphs can be endowed with the structure of a convex cell complex [2], which allows the use of methods from algebraic topology to prove non-existence results concerning homomorphism, in particular colorings [3, 4. Can this be extended to categories? Again, one can generalize the definitions in more than one way, but the generalization of the results, and even more finding interesting further special cases, is open.

\section{References}

[1] J. Adámek, H. Herrlich and G.E. Strecker: Abstract and Concrete Categories: The Joy of Cats, Reprints in Theory and Applications of Categories 17 (2006), 1-507. http://katmat.math.uni-bremen.de/acc/acc.pdf

[2] E. Babson and D.N. Kozlov: Complexes of graph homomorphisms, Isr. J. Math. 152 (2006), 285-312.

[3] E. Babson and D.N. Kozlov: Proof of the Lovász conjecture, Annals of Math. 165 (2007), 965-1007.

[4] E. Babson and D. Kozlov: Topological obstructions to graph colorings, http://lanl .arxiv.org/abs/math.CO/0305300

[5] C. Borgs, J.T. Chayes, L. Lovász, V.T. Sós, and K. Vesztergombi: Convergent Graph Sequences I: Subgraph frequencies, metric properties, and testing, Advances in Math. (2008), 10.1016/j.aim.2008.07.008. 
[6] G. Elek and B. Szegedy: Limits of Hypergraphs, Removal and Regularity Lemmas. A Non-standard Approach, http://arxiv.org/0705.2179

[7] M. Freedman, L. Lovász and A. Schrijver: Reflection positivity, rank connectivity, and homomorphisms of graphs, J. Amer. Math. Soc. 20 (2007), 37-51.

[8] P. Hell and J. Nesetril: Graphs and Homomorphisms, Oxford University Press, 2004.

[9] L. Lovász: Operations with structures, Acta Math. Hung. 18 (1967), 321-328.

[10] L. Lovász: Direct product in locally finite categories, Acta Sci. Math. Szeged 23 (1972), 319-322.

[11] L. Lovász and A. Schrijver: Dual graph homomorphism functions (submitted).

[12] L. Lovász and B. Szegedy: Limits of dense graph sequences, J. Comb. Theory B 96 (2006), 933-957.

[13] L. Lovász and B. Szegedy: The moment problem for 2-variable functions and reflection positive graph parameters (manuscript) http://www.cs.elte.hu/ lovasz/moment.pdf

[14] A. Pultr: Isomorphism types of objects in categories determined by numbers of morphisms, Acta Sci. Math. Szeged 35 (1973), 155-160.

[15] A.A. Razborov: Flag Algebras, Journal of Symbolic Logic, 72 (2007), 1239-1282.

[16] A. Schrijver: Graph invariants in the spin model (manuscript), http://www.cwi.nl/ lex/files/grvm.pdf

[17] B. Szegedy: Edge coloring models and reflection positivity, J. Amer. Math. Soc. 20 (2007), 969-988. 